\title{
Artificial intelligence (AI) assisted fatigue fracture recognition based on morphing and Fully Convolutional Networks
}

\author{
Yetao Lyu ${ }^{1}$, Zi Yang ${ }^{1}$, Hao Liang${ }^{1}$, Beini Zhang ${ }^{2}$, Ming Ge ${ }^{3}$, Rui Liu ${ }^{4}$, Zhefeng Zhang ${ }^{4}$, and \\ Haokun Yang ${ }^{1}$ \\ ${ }^{1}$ Hong Kong Productivity Council \\ ${ }^{2}$ The Hong Kong University of Science and Technology \\ ${ }^{3}$ Hong Kong Industrial Artificial Intelligence and Robotics Centre (FLAIR) Hong Kong \\ People's Republic of China \\ ${ }^{4}$ Institute of Metal Research Chinese Academy of Sciences
}

September 25, 2021

\begin{abstract}
Fatigue fracture is one of the most common metallic component failure cases in manufacturing industries. The observation on fractography can provide the direct evidence for failure analysis. In this study, an image segmentation method based on Fully Convolutional Networks (FCNs) was proposed to figure out the boundary between fatigue crack propagation and fast fracture regions from optical microscope (OM) fractography images. Furthermore, novel morphing-based data augmentation method was adopted to enable few-shot learning of sample images. The proposed framework can successfully segment two categories, namely the crack propagation and fast fracture regions, thus differentiating the boundary of two regions in one image. The artificial intelligence (AI) assisted fatigue analysis architecture can complete the failure analysis procedure in 0.5 second and prove the feasibility of fatigue failure analysis. The segmentation accuracy of developed network achieves $95.4 \%$ for the fatigue crack propagation region, as well as $97.2 \%$ for the fast fracture region, which possesses comparable accuracy to the segmentation results using Mask R-CNN Regional Convolutional Neural Network (Mask R-CNN), one state-of-the-art deep learning networks.
\end{abstract}

\section{Hosted file}

AI+fatigue+analysis full-v11.docx available at https://authorea.com/users/436785/articles/ 538867-artificial-intelligence-ai-assisted-fatigue-fracture-recognition-based-onmorphing-and-fully-convolutional-networks 